\title{
MODELLING OF AN ARTIFICIAL NEURAL NETWORK FOR ELECTRICAL DISCHARGE MACHINING OF HOT PRESSED ZIRCONIUM DIBORIDE-SILICON CARBIDE COMPOSITES
}

\begin{abstract}
Summary
Modelling is carried out to map the relationship between the input process parameters and the output response, considered in the machining process. To represent real-world systems of considerable complexity, an artificial neural network (ANN) model is often utilized to replace the mathematical approximation of the relationship. This paper explains the methodological procedure and the outcome of the ANN modelling process. The percentage of $\mathrm{SiC}$ in the workpiece material, the product of thermal conductivity and the melting point of the tool material, the pulse on time, and the pulse off time are considered as input parameters, while the material removal rate (MRR), the tool wear rate (TWR), roughness, roundness, taper angle and overcut are considered as output responses. The network is trained initially with one neuron in the hidden layer, i.e.,-a 4-4-6 topology is considered for training. In the subsequent phases, the number of hidden neurons in the hidden layer is increased gradually and then the network is tested with two hidden layers with the same number of hidden neurons in the second hidden layer. A feed forward back propagation neural network model with one hidden layer having 35 neurons is found to be the optimum network model (4-35-356). The model has the mean correlation coefficient of 0.92408 .
\end{abstract}

Key words: $\quad$ artificial neural network (ANN), composite, electrical discharge machining (EDM), machining, modelling, silicon carbide ( $\mathrm{SiC})$, zirconium di boride $\left(\mathrm{ZrB}_{2}\right)$

\section{Introduction}

The present manufacturing environment is characterized by complexity, interdisciplinary manufacturing functions and an ever growing demand for new tools and techniques to solve difficult problems. A neural network is used to capture the general relationship between variables of a system that are difficult to relate analytically. Neural network described as a brain metaphor of information processing or as a biologically inspired statistical too1 [22]. It has the capability to learn or to be trained for particular task, its own computational capabilities and the ability to formulate abstractions and generalizations. Neural network has an organization similar to that of a human brain and it is a network made up of processing elements called neurons. Neurons get data from the surrounding neurons, perform some computations and pass the results to other neurons. Connections between the 
neurons have weight associated with them. In the neural network, the knowledge is stored in its interconnection weights in an implicit manner; learning takes place within the system and plays the most important role in the construction of a neural network system. The neural network system learns by determining the interconnection weights from a set of given data [23]. A mathematical model was proposed to analyse the impacts of combustion parameters on pollutant production and combustion process efficiency. The influence of the air excess factor, fuel droplet size, fuel spray angle, and intensity of the swirl of combustion air on the reduction in the amount of $\mathrm{CO}, \mathrm{NO}$, soot, $\mathrm{SOx}$ and in the heat flux released to furnace walls is investigated[16]. Karsaj et al. [7] used a free energy-based formulation incorporating the effect of kinematic hardening. The computational algorithm was also implemented at the material point level of a shell finite element which allows the use of complete threedimensional constitutive laws. Robustness and efficiency of the proposed algorithm are demonstrated by numerical examples. Lela et al. [11] proposed three different models, namely regression analysis (RA), support vector machines (SVM), and Bayesian neural network (BNN), to examines the influence of cutting speed, feed, and depth of cut on surface roughness in the face milling process. All models show a relative prediction error of less than $8 \%$ and the best prediction of surface roughness is made by the BNN model with the average relative prediction error of $6.1 \%$.

The derived mathematical model was validated and tested which enabled the calculation of machining time required for complex cylindrical part production in a given experimental set-up condition. For real-time systems, Bashiri and Miremadi[1] proposed a schedulability condition, that guarantees a desired level of performability in various working conditions. The proposed condition is evaluated on a hard real-time system that employs the rate-monotonic (RM) scheduling algorithm and uses the re-execution mechanism to improve the reliability. Evaluation results reveal that by employing the schedulability condition, the level of performability of the system is always higher than the desired performability.

In the past decade, neural networks have proven to be highly flexible modelling tools with capabilities for learning the mathematical mapping between input variables and output features for nonlinear systems [3]. Lina et al. [13] established the relationship between machining forces and wear of a tool wear made of an aluminium metal matrix composite by using a multiple regression analysis (MRA) and a generalized radial basis function (GRBF) neural network. Liao and Chin [12] used a generalized back-propagation (BP) neural network with two-hidden layers to establish a model for the grinding process. Then, this algorithm, together with the Boltzmann factor, was used to find global optimal settings for the grinding process. It was found that the implemented neural network approach yields a more accurate process model than the regression method. Lee et al. [10] used an abductive network for the modelling of drilling processes; this network was composed of a number of functional nodes which were self-organized to form the optimal network architecture by using a predicted squared error criterion. Ko and Cho [9] introduced Two neural networks: one for estimating the tool wear length, and the other for mapping input and output relations from the experimental data obtained during cutting in the face milling operation. Zuperl [24] et al combined neural networks, fuzzy logic and the particle swarm optimization (PSO) evolutionary strategy in modelling and adaptively controlling the process of ball-end milling. The adaptive control system was developed to control the cutting force and maintaining constant roughness of the surface being milled. A mathematical model of thermo-mechanical processes, such as welding and heat treatment processes, was created by Urevc et al. [21]. In this model, the temperature-dependent thermal and mechanical material parameters are identified from given experimental data obtained for an austenite stainless steel; then, the adequacy of the adopted model and of identified material parameters was shown by simulating numerically a three pass butt welding experiment where good agreement between 
the calculated results and data from the literature was found. Finally annealing of the welded plate to relieve residual stress was simulated numerically. Gjeldum et al. [4] proposed a mathematical model for $M R R$ based on the input parameters such as pulse maximum current, pulse pause time, rotation speed, length of discharge area, and cutting radius.

Rangwala and Dornfeld [15] applied neural networks to integrate information from multiple sensors in order to recognize the occurrence of tool wear in a turning operation. The superior learning and noise suppression abilities of these networks enable high success rates in recognizing tool wear under a range of machining conditions. Tarng et al. [18 and 19] employed back propagation (BP) on the neural networks used for monitoring tool wear and breakage in turning or drilling processes. Cariapa et al. [2] used neural networks in predicting and controlling polishing operations and showed that neural networks with one hidden layer learn faster than those with two hidden layers.

In the electrical discharge machining (EDM) process, both Kao and Tarng [6] and Liu and Tarng [14] employed feed-forward neural networks with hyperbolic tangent functions and adductive networks for the on-line recognition of pulse-types. Based on their results, discharge pulses have been identified and then employed for controlling the EDM machine. Meanwhile, Katz and Naude [8] used back propagation errors in neural networks for improving the geometric shape of EDM products based on the coupling feature design in the EDM process. Tarng et al. [20] utilized a feed forward neural network to associate the cutting parameters with the cutting performance. Subsequently a simulated annealing (SA) algorithm was applied to the neural network to solve the optimal cutting parameters for wire electrical discharge machining (WEDM) process based on a performance index within the allowable working conditions. In this study, the modelling of the effects of pulse on time, pulse off time and tool materials characteristics in the EDM process of $\mathrm{ZrB}_{2}-\mathrm{SiC}$ is carried out using ANNs. The influence of each parameter on machinability behaviour is determined by applying desirability functional analysis (DFA).

\section{Design of experiments}

Workpieces of different volume proportions of $\mathrm{ZrB}_{2}$ and $\mathrm{SiC}(15,20,25$ and $30 \%$ of $\mathrm{SiC}$ with $\mathrm{ZrB}_{2}$ ) are manufactured using the powder metallurgy technique. Electrodes made of tungsten; niobium, tantalum, graphite and titanium are selected for this study. Electronica small sinker $500 \times 300$ series machine was used for experimentation. The specification of this machine is max working current $-35 \mathrm{~A}$, work table size $-500 \times 300 \mathrm{~mm}$, Power supply-3 phase, $415 \mathrm{VAC}, 50 \mathrm{~Hz}$. The experiment is set as a full factorial experiment. Table 1 gives the details of experimental design. Each tool performs nine operations on a particular workpiece. In total, there are 45 operations conducted on each workpiece. MRR, TWR, roughness, roundness, taper angle and overcut are considered as output responses. EDM condition for the experiment is shown in Table 2.

Table 1 Experimental conditions

\begin{tabular}{|l|l|}
\hline \multirow{2}{*}{ Polarity } & Workpiece : Positive \\
\cline { 2 - 2 } & Tool : Negative \\
\hline Open circuit voltage & $100 \mathrm{~V}$ \\
\hline Discharge current & $2 \mathrm{~A}$ \\
\hline Workpiece dimension & $65 \times 65 \times 4.2 \mathrm{~mm}$ \\
\hline Tool diameter & $2 \mathrm{~mm}$ \\
\hline
\end{tabular}


Table 2 Design of experiments

\begin{tabular}{|c|c|c|c|c|c|}
\hline Factor/Level & $\mathbf{1}$ & $\mathbf{2}$ & $\mathbf{3}$ & $\mathbf{4}$ & $\mathbf{5}$ \\
\hline Pulse on $(\mu \mathrm{s})$ & 4 & 7 & 10 & - & - \\
\hline Pulse off $(\mu \mathrm{s})$ & 1 & 3 & 5 & - & - \\
\hline & $85 \% \mathrm{ZrB}-$ & $80 \% \mathrm{ZrB}-$ & $75 \% \mathrm{ZrB}-$ & $70 \% \mathrm{ZrB}-$ & - \\
Workpiece $(\mathrm{No})$ & $15 \% \mathrm{SiC}_{2}(1)$ & $20 \% \mathrm{SiC}_{2}(2)$ & $25 \% \mathrm{SiC}(3)$ & $30 \% \mathrm{SiC}(4)$ & - \\
\hline Tool $(\mathrm{No})$ & Graphite $(1)$ & Titanium(2) & Niobium $(3)$ & Tantalum $(4)$ & Tungsten(5) \\
\hline
\end{tabular}

In this study, five tool materials are selected for experiments. The tools of $2 \mathrm{~mm}$ in diameter, made from different materials were fabricated using different techniques like wire cut EDM, diamond grinding and extrusion process. Fig.3.1 shows a photographic image of the tools.

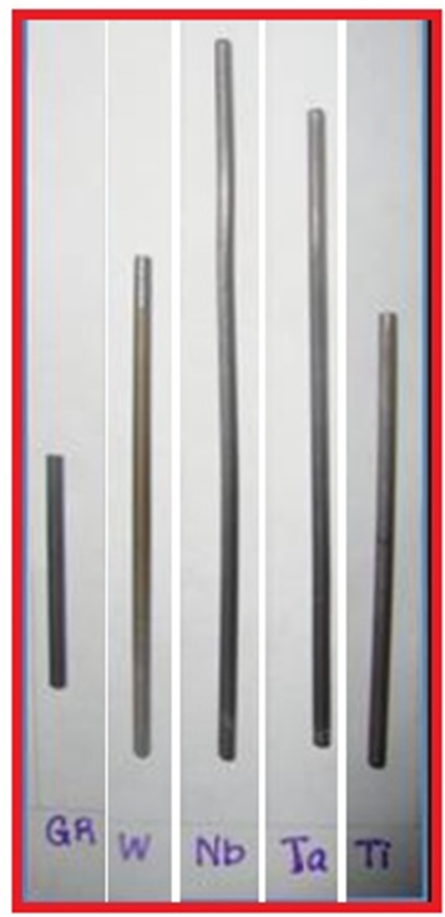

Fig. 1 Photographic image of the tools

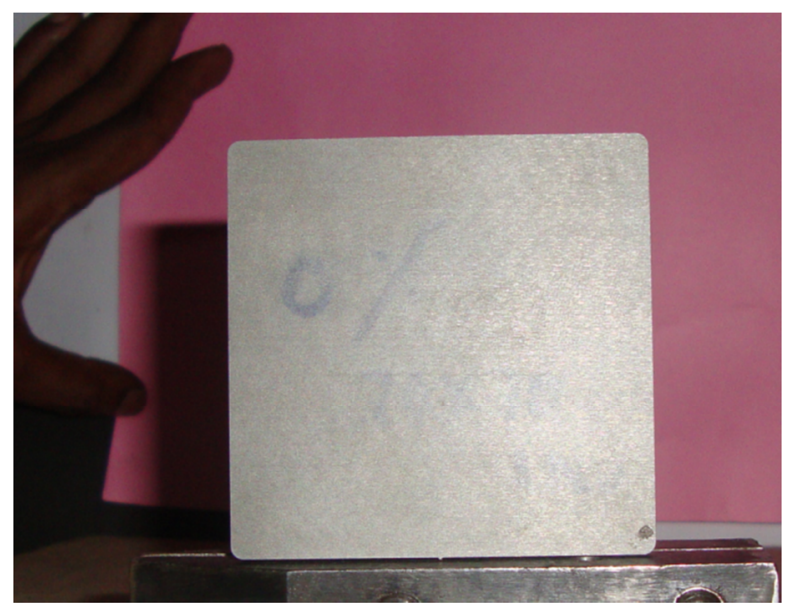

Fig. 2 Workpiece after grinding and square cut

$\mathrm{ZrB}_{2}-\mathrm{SiC}$ composites were hot pressed in an organ atmosphere to a disc of $100 \mathrm{~mm}$ in diameter and thickness of $5 \mathrm{~mm}$. The temperature during pressing was maintained at $2000^{\circ} \mathrm{C}$ with a pressure of $30 \mathrm{MPa}$. The compaction was done for a period of $60 \mathrm{~min}$. The hot pressed disc surfaces did not have uniform surfaces and may have contained graphite particles. In order to overcome that problem, these discs were subjected to the sand blasting process. After that, the discs were subjected to the diamond load grinding to obtain coplanar surfaces. A square block of $65 \mathrm{~mm}$ was engraved from the disc using the WEDM process to facilitate the clamping positional accuracy of the hole. Fig.2 shows a square shaped workpiece. 


\section{Output responses}

MRR is calculated based on the weight by using equation 1. Machining time was measured using a stop watch.

$M R R=\frac{\text { Weight of workpiece before machining }- \text { Weight of workpiece after machining }}{\text { Machining time }}$.

There are four methods known to evaluate the tool wear rate by means of measuring weight, shape, length, and total volume. In this study, the tool wear rate is evaluated by means of weight measurement (equation 2).

$T W R=\frac{\text { Weight of tool before machining-Weight of tool after machining }}{\text { Machining time }}$

Roughness, roundness, taper angle and overcut are measured by means of image processing techniques. The procedure of measuring these parameters is explained by Sivasankar et al [17].

\section{Back propagation neural network}

The back propagation algorithm (BP) has made it possible to design multi-layer neural networks for numerous applications, such as adaptive control, classification of sonar targets, stock market prediction and speech recognition. Also, back propagation neural network (BPNN) has the advantage of quick response and high learning accuracy. Thus, an ANN with the back propagation algorithm has been applied here to model the output responses related to input parameters of the EDM process of $\mathrm{ZrB}_{2}-\mathrm{SiC}$ composites. The algorithm of the back propagation program is shown in Fig. 3.

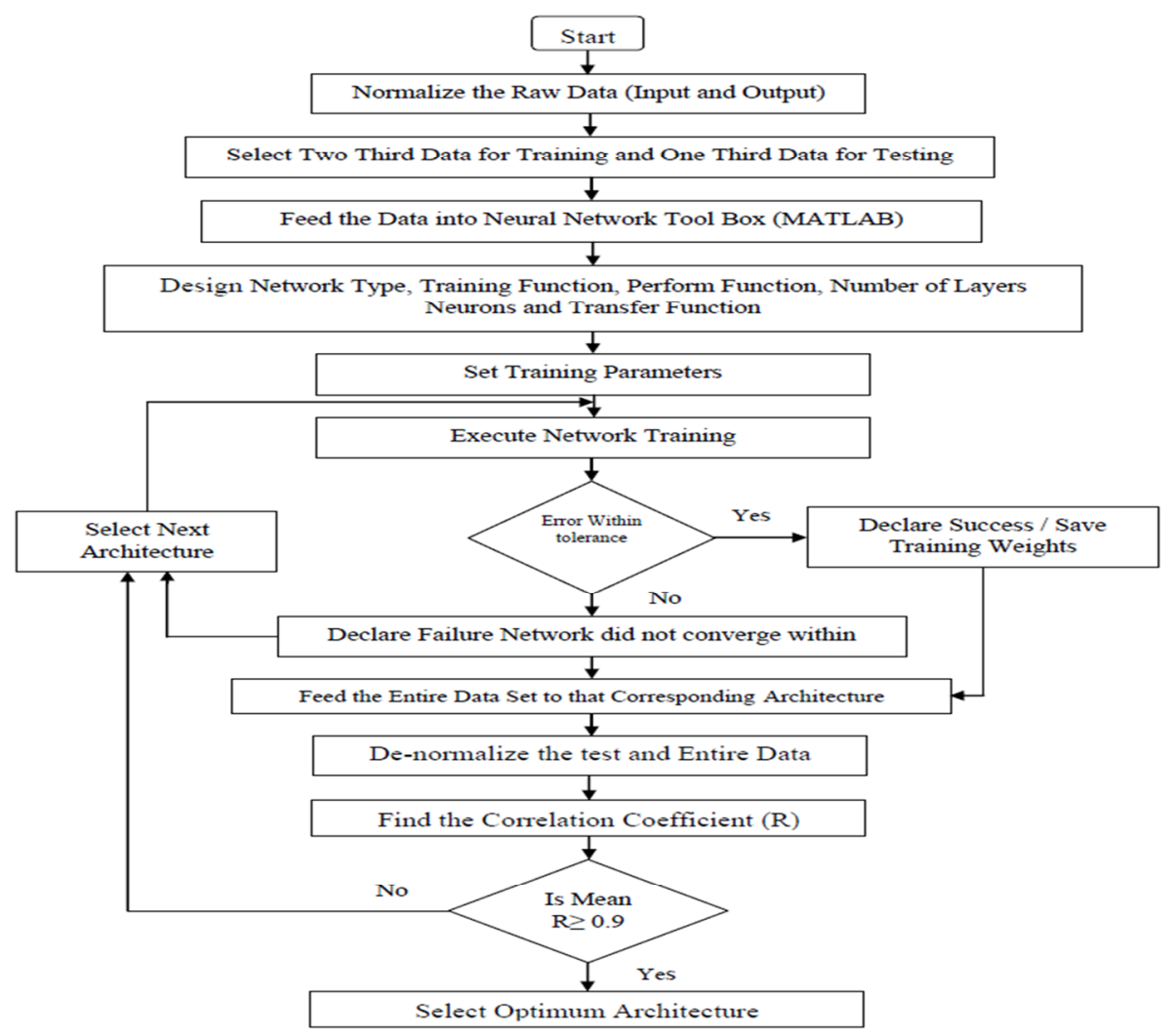

Fig. 3 Flow diagram for the selection of optimum ANN architecture 


\section{Model description}

In the development of a multi-layer neural network model, several decisions regarding the number of neuron(s) in the input layer, the number of hidden layer(s), the number of neuron(s) in the hidden layer(s), and the number of neuron(s) in the output layer and optimum architectures have to be made. Based on the experimental condition, the pulse on time, the pulse off time, the tool material and the vol. \% $\mathrm{SiC}$ are given as input parameters to the present ANN model. The output parameters are MRR, TWR, roughness, roundness, overcut and taper angle. The input/output dataset of the model is illustrated schematically in Fig. 4.

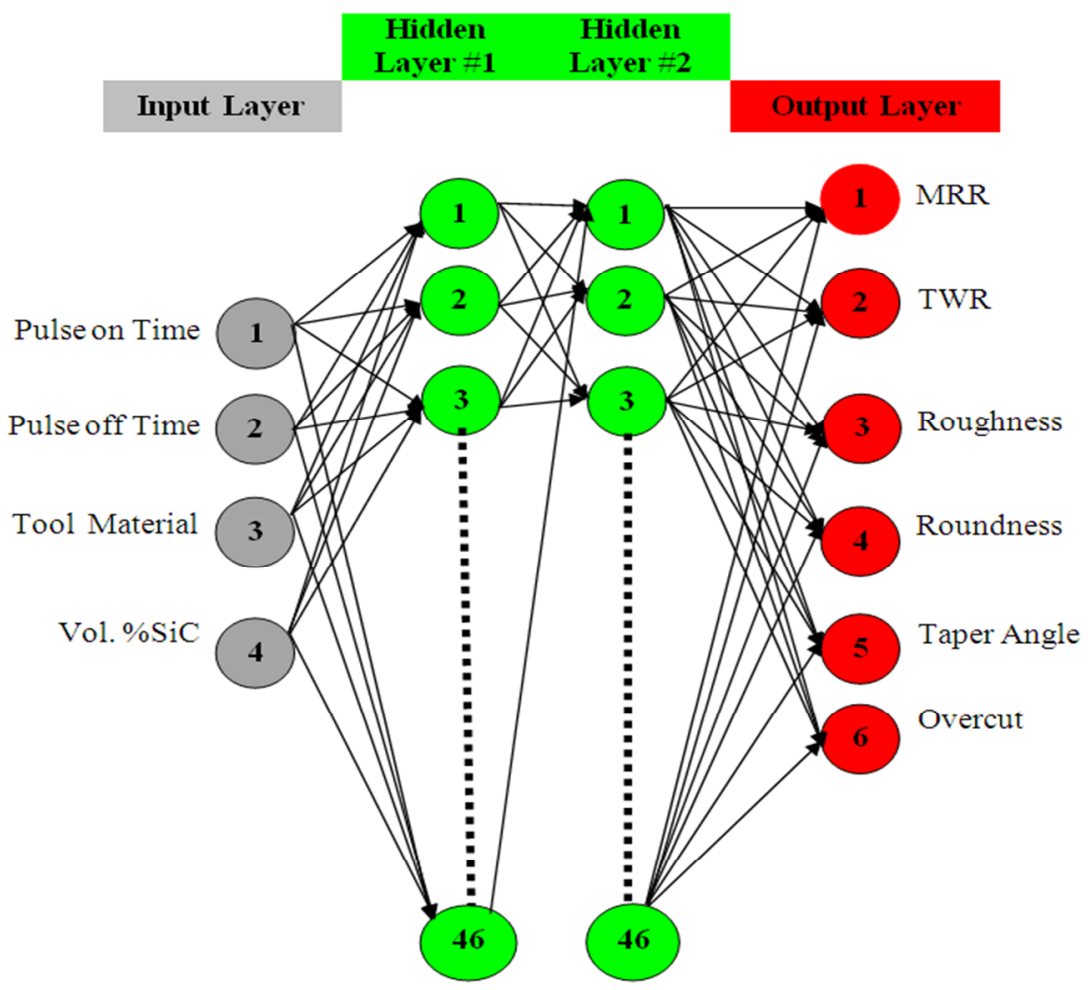

Fig. 4 Schematic illustration of the ANN model for the EDM of $\mathrm{ZrB}_{2}$-SiC Composites

\section{Neural network design and training}

The generalization capability of the neural network mainly depends on: (i) the selection of appropriate input/output parameters of the system, (ii) the distribution of the dataset and (iii) the format of the presentation of the dataset to the network. In this study, the total number of experimental results is $180(45 \times 6=180$ total $)$ datasets among which 150 datasets (more than two-thirds) have been considered for training, and 30 datasets for testing. Before training the network, the input/output datasets were normalized within the range of \pm 1 .

\section{Data normalization}

Higher value input variables may tend to suppress the influence of smaller value ones. To overcome this problem, the neural networks were trained with the normalized input data, leaving it to the network to learn the weights associated with the connections emanating from these inputs. The raw data to be used by neural networks were scaled in the range 1 to +1 to minimize the effects of magnitude between inputs and also to aid the backpropagation of the learning algorithm. The normalized values $\left(X_{n}\right)$ for each raw input/output dataset $\left(d_{i}\right)$ were calculated using equation 3 


$$
X_{n}=\frac{2\left(d_{i}-d_{\min }\right)}{\left(d_{\max }-d_{\min }\right)}-1
$$

where $d_{\max }$ and $d_{\min }$ are the maximum and the minimum values of raw data.

The standard multi-layer feed forward backpropagation hierarchical neural networks were designed in the MATLAB (version 7.10.0) Neural Network Toolbox. The networks consist of three layers: the input, the hidden layer, and the output layer. Now, the designed network has four input neurons and six output neurons. Kumaran et al. (2010) gave a step by step description of how neural network works. In the network, each neuron receives the total input from all of the neurons in the proceeding layer as expressed in equation 4.

$$
n e t_{j}=\sum_{i} W_{i j}^{n}\left(X_{i}\right)^{n-1}
$$

where $n e t_{j}$ is the total or net input, $X_{i}^{n}$ is the output of the node $\mathrm{j}$ in the nth layer, and $W_{i j}^{n}$ represents the weights from node $\mathrm{i}$ in the $(n-1)$ th layer to node $j$ in the nth layer. A neuron in the network produces its input by processing the net input through an activation (transfer) function which is usually nonlinear. There are several types of activation functions used for BP. However, the most often used function is the tan-sigmoid transfer function which is assigned to hidden layer(s) for processing the inputs as in the equation shown below (equation 5). Purelin is a transfer function calculating the output of a hidden layer from its net input which is assigned to the output layer as in equation 6 .

$$
\begin{aligned}
& f_{(x)}=\left(\frac{e^{x}-e^{-x}}{e^{x}+e^{-x}}\right)-1 \text { range }(-1,1) \\
& f_{(x)}=\frac{2}{\left(1+e^{-x}\right)} \text { range }(-1,1)
\end{aligned}
$$

The weights are dynamically updated using the BP algorithm. The network has been trained with the Levenberg-Marquardt algorithm. This training algorithm is selected due to its high accuracy in a similar function approximation. In order to estimate the performance of the network, the mean square error (MSE), which is obtained using equation 7, has been used.

$$
E_{p}=\sum_{p=1}^{p} \sum_{k=1}^{k}\left(d_{p k}-o_{p k}\right)
$$

where $d_{p k}$ and $o_{p k}$ are the desired and the calculated output for the $k^{\text {th }}$ response, respectively. The ' $k$ ' denotes the number of neurons in the network output and ' $p$ ' is the total number of instances (epochs). The number of iterations (epochs) to be executed is an important parameter in the case of the BPNN training. After training, the network has been denormalized and compared with the experimental data. The denormalized values $\left(x_{i}\right)$ for each raw output dataset was calculated as per equation 8 .

$$
X_{i}=\frac{\left(X_{n}+1\right)\left(d_{\max }-d_{\min }\right)}{2}+d_{\min }
$$

where $d_{\max }$ and $d_{\min }$ are the maximum and minimum values of raw data.

For testing the prediction ability of the model, the prediction error $(P E)$ in each output node has been calculated as per equation 9.In order to determine the optimal architecture, the 
mean PE is calculated for converged architectures, and the one that shows a higher correlation coefficient value and the minimum average PE is selected as the optimal architecture.

$$
\mathrm{PE} \%=\frac{\text { Actual value }- \text { Predicted value }}{\text { Actual value }} \times 100
$$

\section{Testing and performance of the BPNN model}

An ANN with the BPNN algorithm has been adopted here for modelling. The optimization of the layer and the number of neurons in the network is done by evaluating the performance of different architectures. Twenty five different architectures are evaluated and the best performance architecture is the one with four layers with 4-35-35-6 neurons in the layers. The performance capability of each network has been examined based on the correlation coefficient and the convergence of entire dataset within a specified error range between the network predictions and the experimental values using the test and the entire dataset. In order to determine the optimum structure of neural network, the rate of error convergence is checked by changing the number of hidden neurons and the number of hidden layers. In Table 3, one can note that the network with 35 neurons in each hidden layer has produced the best performance for each of the output parameters (4-35-35-6), because the mean correlation observed is high $(92.408 \%)$. The correlation coefficient $(R)$ for each response is calculated by using the following equation (10)

$$
R=\frac{n \sum x y-\sum x \sum y}{\sqrt{\left(n \sum x^{2}-\left(\sum x\right)^{2}\right) \times\left(n \sum y^{2}-\left(\sum y\right)^{2}\right)}}
$$

\begin{tabular}{|c|c|c|c|}
\hline $\begin{array}{l}\text { Sl. } \\
\text { No. }\end{array}$ & $\begin{array}{l}\text { Network } \\
\text { Architecture }\end{array}$ & $\begin{array}{c}\text { Performance } \\
\text { (M.S.E) }\end{array}$ & $\begin{array}{c}\text { Status of } \\
\text { convergence }\end{array}$ \\
\hline 1 & $4-4-6$ & 0.0365 & Not converged \\
\hline 2 & $4-8-6$ & 0.0284 & Not converged \\
\hline 3 & $4-12-6$ & 0.0453 & Not converged \\
\hline 4 & $4-16-6$ & 0.0432 & Not converged \\
\hline 5 & $4-20-6$ & 0.0437 & Not converged \\
\hline 6 & $4-24-6$ & 0.0307 & Not converged \\
\hline 7 & $4-28-6$ & 0.0234 & Not converged \\
\hline 8 & $4-32-6$ & 0.0321 & Not converged \\
\hline 9 & $4-36-6$ & 0.0213 & Not converged \\
\hline 10 & $4-40-6$ & 0.0546 & Not converged \\
\hline 11 & $4-44-6$ & 0.0612 & Not converged \\
\hline 12 & $4 .-48-6$ & 0.0232 & Not converged \\
\hline 13 & $4 .-52-6$ & 0.0242 & Not converged \\
\hline 14 & $4-10-10-6$ & 0.0536 & Not converged \\
\hline 15 & $4-15-15-6$ & 0.0653 & Not converged \\
\hline 16 & 4-18-18-6 & 0.0432 & Not converged \\
\hline 17 & $4-20-20-6$ & 0.0354 & Not converged \\
\hline 18 & $4-22-22-6$ & 0.0303 & Not converged \\
\hline 19 & $4-25-25-6$ & 0.0213 & Not converged \\
\hline 20 & $4-28-28-6$ & 0.0201 & Converged \\
\hline 21 & $4-32-32-6$ & 0.0165 & Converged \\
\hline 22 & $4-35-35-6$ & 0.0124 & Converged \\
\hline 23 & $4-40-40-6$ & 0.0231 & Converged \\
\hline 24 & $4-44-44-6$ & 0.0452 & Converged \\
\hline 25 & $4-46-46-6$ & 0.0354 & Converged \\
\hline
\end{tabular}

Table 3 Performance value and the status of convergence of different network architecture 
Based on the optimized network parameters, an ANN model has been developed to predict the machinability behaviour of $\mathrm{ZrB}_{2}-\mathrm{SiC}$ composite in terms of MRR, TWR, roughness, roundness, taper angle and overcut. Table 3 shows the performance value and the status of convergence of different networks architectures. Out of the 25 networks, the 4-35-35-6 architecture shows better performance than all other networks. The architectures, with more than 40 neurons in the hidden layers also show lower performance values than the 4-35-35-6 network architecture. The performance capability of the converged network has been examined based on the correlation coefficient of the entire dataset within a specified error range between the network predictions and the experimental values using the test and the entire dataset. The results obtained for the architecture are shown in Table 4.The actual values of the tested values of the response are shown in Table 5, while the predicted values of the optimal network (4-35-35-6) and the prediction error from the experimental results are shown in Table 6. The mean prediction error obtained for the entire dataset by means of optimal network was 2.14 percent.

Table 4 Mean Correlation coefficient between the network predictions and the experimental values

\begin{tabular}{|c|c|c|c|c|c|c|}
\hline $\begin{array}{c}\text { Sl. } \\
\text { No. }\end{array}$ & 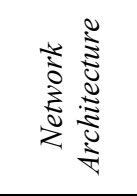 & 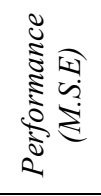 & 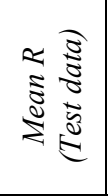 & 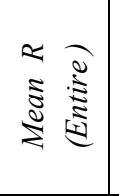 & 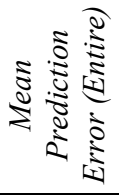 & 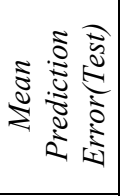 \\
\hline 1 & $4-4-6$ & 0.0365 & 0.762 & 0.794 & 0.5031 & 5.24 \\
\hline 2 & $4-8-6$ & 0.0284 & 0.7234 & 0.764 & 0.347 & 2.548 \\
\hline 3 & $4-12-6$ & 0.0453 & 0.7844 & 0.713 & 0.3354 & 2.824 \\
\hline 4 & $4-16-6$ & 0.0432 & 0.7987 & 0.8000 & 0.542 & 1.71 \\
\hline 5 & $4-20-6$ & 0.0437 & 0.7485 & 0.738 & 0.687 & 2.1 \\
\hline 6 & $4-24-6$ & 0.0307 & 0.7862 & 0.800 & 0.544 & 2.68 \\
\hline 7 & $4-28-6$ & 0.0234 & 0.8341 & 0.812 & 0.354 & 6.84 \\
\hline 8 & $4-32-6$ & 0.0321 & 0.8536 & 0.832 & 0.213 & 2.14 \\
\hline 9 & $4-35-6$ & 0.0213 & 0.9015 & 0.890 & 0.176 & 5.24 \\
\hline 10 & $4-40-6$ & 0.0546 & 0.8748 & 0.843 & 0.321 & 2.548 \\
\hline 11 & $4-44-6$ & 0.0612 & 0.8458 & 0.8140 & 0.425 & 2.824 \\
\hline 12 & $4-48-6$ & 0.0232 & 0.8920 & 0.824 & 0.325 & 1.71 \\
\hline 13 & $4-52-6$ & 0.0242 & 0.8632 & 0.814 & 0.432 & 2.1 \\
\hline 14 & $4-10-10-6$ & 0.0536 & 0.7909 & 0.783 & 0.635 & 2.14 \\
\hline 15 & $4-15-15-6$ & 0.0653 & 0.7385 & 0.760 & 0.324 & 5.24 \\
\hline 16 & $4-18-18-6$ & 0.0432 & 0.8002 & 0.792 & 0.432 & 2.548 \\
\hline 17 & $4-20-20-6$ & 0.0354 & 0.8109 & 0.783 & 0.542 & 2.824 \\
\hline 18 & $4-22-22-6$ & 0.0303 & 0.8408 & 0.8209 & 0.300 & 1.71 \\
\hline 19 & $4-25-25-6$ & 0.0213 & 0.8943 & 0.87601 & 0.323 & 2.1 \\
\hline 20 & $4-28-28-6$ & 0.0201 & 0.9134 & 0.89501 & 0.210 & 2.68 \\
\hline 21 & $4-32-32-6$ & 0.0165 & 0.9232 & 0.91232 & 0.243 & 6.84 \\
\hline 22 & $4-35-35-6$ & 0.0124 & 0.9137 & 0.92408 & 0.321 & 2.14 \\
\hline 23 & $4-40-40-6$ & 0.0231 & 0.8901 & 0.90341 & 0.314 & 2.548 \\
\hline 24 & $4-44-44-6$ & 0.0452 & 0.8530 & 0.87632 & 0.241 & 2.824 \\
\hline 25 & $4-46-46-6$ & 0.0354 & 0.8322 & 0.85352 & 0.310 & 1.71 \\
\hline
\end{tabular}

Table 5 The actual value of responses for the tested input

\begin{tabular}{|c|c|c|c|c|c|c|}
\hline Ex.No & MRR & TWR & Roughness & Roundness & Taper angle & overcut \\
\hline 1 & 1.045899 & 1.018797 & 7.065147 & 84.85563 & 5.681909 & 0.46992 \\
\hline 2 & 1.085849 & 1.070194 & 6.53382 & 100.8786 & 4.994312 & 0.624187 \\
\hline 3 & 0.967932 & 1.00063 & 5.232679 & 83.48449 & 5.279503 & 0.598114 \\
\hline 4 & 0.780454 & 1.085435 & 8.047817 & 79.33548 & 5.581564 & 0.634101 \\
\hline 5 & 0.553376 & 1.083306 & 7.521314 & -179.099 & 5.571762 & 0.480875 \\
\hline 6 & 0.349042 & 1.012289 & 7.623175 & 39.60496 & 6.598838 & 0.742319 \\
\hline 7 & 0.417971 & 1.046649 & 6.234103 & 66.94923 & 6.462446 & 0.741111 \\
\hline
\end{tabular}


Modelling of an Artificial Neural Network for Electrical Discharge Machining of Hot Pressed Zirconium Diboride-Silicon Carbide Composites

\begin{tabular}{|c|c|c|c|c|c|c|}
\hline Ex.No & $M R R$ & $T W R$ & Roughness & Roundness & Taper angle & overcut \\
\hline 8 & 2.181339 & 1.005055 & 7.886682 & 132.2382 & 2.707276 & 0.397083 \\
\hline 9 & 0.973873 & 1.014343 & 9.325849 & 135.8369 & 1.136477 & 0.181976 \\
\hline 10 & 0.976746 & 1.001733 & 9.028151 & 118.0327 & 3.23885 & 0.211941 \\
\hline 11 & 1.657861 & 1.005864 & 12.80665 & 123.2341 & 1.230785 & 0.145051 \\
\hline 12 & 0.459418 & 1.008207 & 7.280956 & 109.407 & 3.852926 & 0.15913 \\
\hline 13 & 1.947682 & 1.012802 & 6.377133 & 407.0044 & 3.515488 & 0.279902 \\
\hline 14 & 0.483159 & 1.001031 & 5.629013 & 136.7531 & 5.350721 & 0.599603 \\
\hline 15 & 0.543978 & 1.066087 & 14.54474 & 203.1373 & 4.33298 & 0.264018 \\
\hline 16 & 0.896047 & 1.018174 & 9.590224 & 72.72493 & 5.491963 & 0.72368 \\
\hline 17 & 0.715599 & 1.033229 & 7.114989 & 70.67075 & 5.730673 & 0.6244 \\
\hline 18 & 0.339785 & 1.071455 & 7.581819 & 56.19326 & 6.676842 & 0.76019 \\
\hline 19 & 2.207994 & 1.003442 & 9.125514 & 129.7458 & 2.504823 & 0.297056 \\
\hline 20 & 0.939778 & 1.015785 & 9.32216 & 131.849 & 3.362519 & 0.426932 \\
\hline 21 & 0.427303 & 1.005561 & 6.778119 & 103.8383 & 2.981467 & 0.200074 \\
\hline 22 & 0.554861 & 1.00003 & 13.15121 & 101.0213 & 3.441843 & 0.251972 \\
\hline 23 & 1.82323 & 1.00841 & 6.776501 & 154.4601 & 4.155108 & 0.236064 \\
\hline 24 & 0.485076 & 1.01147 & 5.796068 & 153.4353 & 5.347471 & 0.604555 \\
\hline 25 & 0.529735 & 1.002335 & 8.403999 & 232.8799 & 5.384952 & 0.334023 \\
\hline 26 & 0.369114 & 1.00013 & 13.09564 & 186.4577 & 5.252543 & 0.387108 \\
\hline 27 & 1.030794 & 1.060648 & 6.386874 & 78.93812 & 6.026337 & 0.523916 \\
\hline 28 & 0.862509 & 1.044027 & 7.68026 & 86.48564 & 3.003193 & 0.53217 \\
\hline 29 & 0.654967 & 1.062575 & 8.705852 & 108.221 & 2.467499 & 0.195002 \\
\hline 30 & 0.339989 & 1.033229 & 7.271856 & 172.068 & 6.582019 & 0.844152 \\
\hline 31 & 0.456973 & 1.049395 & 7.927389 & 187.5236 & 2.689895 & 0.584988 \\
\hline 32 & 1.794957 & 1.004349 & 6.22574 & 63.86913 & 3.054973 & 0.11798 \\
\hline 33 & 2.170367 & 1.011061 & 5.203064 & 177.199 & 1.718313 & 0.079963 \\
\hline 34 & 1.033762 & 1.060648 & 6.162325 & 44.1092 & 1.274006 & 0.190038 \\
\hline 35 & 0.841712 & 1.014652 & 7.56707 & 114.1762 & 2.153177 & 0.202127 \\
\hline 36 & 0.716745 & 1.033229 & 7.552726 & 20.5633 & 4.492675 & 0.190148 \\
\hline 37 & 1.550319 & 1.009428 & 6.461773 & 132.6367 & 5.948445 & 0.166151 \\
\hline 38 & 0.433078 & 1.049395 & 5.662207 & 142.1318 & 6.441709 & 0.143225 \\
\hline 39 & 1.847393 & 1.001934 & 4.868083 & 461.6762 & 1.8938 & 0.418941 \\
\hline 40 & 1.120359 & 1.01147 & 7.551064 & 124.967 & 5.900054 & 0.430125 \\
\hline 41 & 1.776164 & 1.060648 & 8.274899 & 271.3755 & 5.734346 & 0.272521 \\
\hline 42 & 1.229525 & 1.062575 & 9.499401 & 130.8801 & 2.426718 & 0.365596 \\
\hline 43 & 0.9091 & 1.070194 & 8.115488 & 130.267 & 4.321396 & 0.23652 \\
\hline 44 & 0.679081 & 1.083424 & 5.427597 & 371.3116 & 3.641154 & 0.168998 \\
\hline 45 & 1.017651 & 1.012494 & 8.284349 & 134.7461 & 3.376914 & 0.286051 \\
\hline 46 & 0.551713 & 1.032908 & 5.389261 & 132.1984 & 3.157726 & 0.479038 \\
\hline 47 & 1.22927 & 1.012494 & 5.374818 & 135.1184 & 3.416941 & 0.480019 \\
\hline 48 & 0.603142 & 1.02046 & 6.024034 & 96.28669 & 4.463554 & 0.419141 \\
\hline 49 & 2.0704 & 1.02622 & 7.849264 & 141.6982 & 1.596625 & 0.593089 \\
\hline 50 & 2.057111 & 1.010959 & 10.05901 & 144.5325 & 2.056398 & 0.62647 \\
\hline 51 & 1.890865 & 1.036871 & 5.78474 & 95.50459 & 6.381973 & 0.342246 \\
\hline 52 & 1.172742 & 1.008919 & 11.45136 & 110.8623 & 5.7144 & 0.063991 \\
\hline 53 & 0.901901 & 1.001332 & 5.952797 & 191.7697 & 5.896619 & 0.340065 \\
\hline 54 & 1.383536 & 1.012494 & 8.81219 & 83.25519 & 0.650336 & 0.498835 \\
\hline 55 & 0.767173 & 1.00053 & 4.913989 & 90.19084 & 1.242525 & 0.782666 \\
\hline 56 & 0.509131 & 1.019212 & 12.73368 & 81.32699 & 6.250367 & 0.157848 \\
\hline 57 & 2.066277 & 1.004652 & 5.362898 & 221.1032 & 0.360596 & 0.383126 \\
\hline 58 & 1.508088 & 1.060648 & 5.096977 & 81.41493 & 3.120982 & 0.559439 \\
\hline 59 & 0.310935 & 1.036871 & 9.542096 & 153.7442 & 3.487626 & 0.503136 \\
\hline 60 & 1.898994 & 1.01942 & 5.014154 & 109.288 & 4.114316 & 0.336973 \\
\hline
\end{tabular}


Modelling of an Artificial Neural Network

for Electrical Discharge Machining of Hot Pressed

Zirconium Diboride-Silicon Carbide Composites

Table 6 Response values obtained by the optimal network and prediction error

\begin{tabular}{|c|c|c|c|c|c|c|c|c|c|c|c|c|}
\hline Ex.No & $M R R$ & P.E & $T W R$ & P.E & Roughness & $P . E$ & Roundness & P.E & Taperangle & $P . E$ & Overcut & $P . E$ \\
\hline 1 & 1.045 & 0.086 & \begin{tabular}{|l|l|}
0.117 \\
\end{tabular} & 1.845 & 7.111 & -0.649 & \begin{tabular}{|l|}
91.751 \\
\end{tabular} & -8.126 & 5.681 & 0.016 & 0.470 & -0.017 \\
\hline 2 & 1.087 & -0.106 & 0.117 & 6.559 & 6.361 & 2.645 & 110.687 & -9.723 & 4.961 & 0.667 & 0.624 & 0.030 \\
\hline 3 & 0.968 & -0.007 & \begin{tabular}{|l|l|}
0.117 \\
\end{tabular} & 0.063 & 5.268 & -0.675 & 92.050 & -10.26 & 5.270 & 0.180 & 0.598 & 0.019 \\
\hline 4 & 0.781 & -0.070 & 0.117 & 7.871 & 8.045 & 0.035 & 73.567 & 7.271 & 5.556 & 0.458 & 0.634 & 0.016 \\
\hline 5 & 0.553 & 0.068 & 0.117 & 7.690 & 7.146 & 4.990 & 114.444 & 163.9 & 5.558 & 0.247 & 0.480 & 0.182 \\
\hline 6 & 0.349 & 0.012 & 0.117 & 1.214 & 7.405 & 2.862 & 41.786 & -5.507 & 6.596 & 0.043 & 0.742 & 0.043 \\
\hline 7 & 0.418 & -0.007 & \begin{tabular}{|l|l|}
0.117 \\
\end{tabular} & 4.457 & 6.250 & -0.255 & 64.288 & 3.975 & 6.466 & - & 0.741 & 0.015 \\
\hline 8 & 2.184 & -0.122 & \begin{tabular}{|l|l|}
0.117 \\
\end{tabular} & 0.503 & 7.781 & 1.340 & 131.138 & 0.832 & 2.720 & - & 0.398 & -0.231 \\
\hline 9 & 0.974 & -0.013 & 0.117 & 1.414 & 9.321 & 0.052 & 141.106 & -3.879 & 1.137 & - & 0.182 & -0.013 \\
\hline 10 & 0.977 & -0.026 & \begin{tabular}{|l|l|}
0.117 \\
\end{tabular} & 0.173 & 9.246 & -2.413 & 91.794 & 22.23 & 3.252 & - & 0.212 & -0.028 \\
\hline 11 & 1.660 & -0.129 & 0.117 & 0.583 & 13.74 & \begin{tabular}{|l|}
-7.288 \\
\end{tabular} & 113.384 & 7.993 & 1.213 & 1.445 & 0.145 & 0.035 \\
\hline 12 & 0.459 & 0.091 & \begin{tabular}{|l|l|}
0.117 \\
\end{tabular} & 0.814 & 7.046 & 3.227 & 69.922 & 36.09 & 3.873 & - & 0.159 & 0.082 \\
\hline 13 & 1.947 & 0.035 & 0.117 & 1.264 & 5.988 & 6.102 & 414.245 & -1.779 & 3.499 & 0.469 & 0.280 & -0.035 \\
\hline 14 & 0.484 & -0.174 & 0.117 & 0.103 & 5.628 & 0.018 & 255.277 & -86.67 & 5.371 & - & 0.601 & -0.233 \\
\hline 15 & 0.544 & -0.004 & \begin{tabular}{|l|l|}
0.117 \\
\end{tabular} & 6.199 & 14.80 & -1.755 & 206.422 & -1.617 & 4.327 & 0.138 & 0.264 & 0.007 \\
\hline 16 & 0.898 & -0.218 & 0.117 & 1.785 & 9.551 & 0.409 & 81.961 & -12.70 & 5.460 & 0.582 & 0.723 & 0.094 \\
\hline 17 & 0.716 & -0.056 & \begin{tabular}{|l|l|}
0.117 \\
\end{tabular} & 3.216 & 7.258 & -2.010 & 71.687 & -1.438 & 5.725 & 0.099 & 0.624 & 0.064 \\
\hline 18 & 0.339 & 0.231 & 0.117 & 6.669 & 7.221 & 4.759 & 40.358 & 28.18 & 6.693 & - & 0.760 & 0.025 \\
\hline 19 & 2.207 & 0.045 & 0.117 & 0.343 & 8.918 & 2.274 & 135.164 & -4.176 & 2.506 & - & 0.297 & 0.019 \\
\hline 20 & 0.941 & -0.130 & 0.117 & 1.554 & 9.400 & -0.835 & 110.529 & 16.17 & 3.368 & - & 0.427 & -0.016 \\
\hline 21 & 0.426 & 0.305 & 0.117 & 0.553 & 6.629 & 2.200 & 95.741 & 7.798 & 2.960 & 0.720 & 0.200 & 0.037 \\
\hline 22 & 0.555 & -0.025 & 0.117 & 0.003 & 14.24 & -8.279 & 106.929 & -5.848 & 3.450 & - & 0.252 & -0.011 \\
\hline 23 & 1.832 & -0.481 & 0.117 & 0.834 & 6.688 & 1.306 & 341.048 & -120.8 & 4.149 & 0.147 & 0.236 & 0.027 \\
\hline 24 & 0.483 & 0.428 & \begin{tabular}{|l|l|}
0.117 \\
\end{tabular} & 1.134 & 5.607 & 3.262 & 257.894 & -68.08 & 5.371 & - & 0.606 & -0.239 \\
\hline 25 & 0.530 & -0.050 & 0.117 & 0.233 & 8.305 & 1.178 & 125.499 & 46.11 & 5.422 & - & 0.334 & 0.007 \\
\hline 26 & 0.369 & 0.031 & \begin{tabular}{|l|l|}
0.117 \\
\end{tabular} & 0.013 & 13.48 & -2.935 & 131.173 & 29.65 & 5.261 & - & 0.387 & 0.028 \\
\hline 27 & 1.031 & -0.020 & 0.117 & 5.718 & 6.279 & 1.689 & 77.267 & 2.117 & 6.027 & - & 0.524 & -0.016 \\
\hline 28 & 0.862 & 0.059 & 0.117 & 4.217 & 7.552 & 1.670 & 82.906 & 4.139 & 3.038 & - & 0.532 & 0.032 \\
\hline 29 & 0.655 & -0.005 & 0.117 & 5.889 & 8.684 & 0.251 & 117.949 & -8.989 & 475 & - & 195 & 0.001 \\
\hline 30 & 0.339 & 0.291 & 0.117 & 3.216 & 7.131 & 1.937 & 43.189 & 125.1 & 682 & - & 0.844 & 0.018 \\
\hline 31 & 0.457 & -0.006 & 0.117 & 4.707 & 8.197 & -3.401 & 185.894 & 0.869 & 2.702 & - & 0.585 & -0.002 \\
\hline 32 & 1.793 & 0.109 & 0.117 & 0.433 & 6.175 & 0.815 & 60.166 & 5.798 & 3.047 & 0.261 & 0.118 & -0.017 \\
\hline 33 & 2.169 & 0.063 & \begin{tabular}{|l|l|}
0.117 \\
\end{tabular} & 1.094 & 5.040 & 3.134 & 183.906 & -3.785 & 1.719 & - & 0.080 & -0.046 \\
\hline 34 & 1.034 & -0.023 & 0.117 & 5.718 & 6.049 & 1.839 & 45.129 & -2.312 & 1.275 & - & 0.190 & 0.020 \\
\hline 35 & 0.843 & -0.153 & \begin{tabular}{|l|l|}
0.117 \\
\end{tabular} & 1.444 & 7.562 & 0.067 & 106.161 & 7.020 & 2.158 & - & 0.202 & 0.063 \\
\hline 36 & 0.716 & 0.104 & 0.117 & 3.216 & 7.592 & -0.520 & 60.744 & 395.4 & 4.465 & 0.616 & 0.190 & 0.078 \\
\hline 37 & 1.567 & -1.076 & 0.117 & 0.934 & 6.093 & 5.707 & 250.166 & -88.61 & 5.956 & - & 0.166 & 0.091 \\
\hline 38 & 0.433 & 0.018 & 0.117 & 4.707 & 5.722 & -1.056 & 86.274 & 39.30 & 6.441 & 0.011 & 0.143 & 0.157 \\
\hline 39 & 1.849 & -0.087 & \begin{tabular}{|l|l|}
0.117 \\
\end{tabular} & \begin{tabular}{|l|l|}
0.193 \\
\end{tabular} & 4.742 & 2.590 & 444.368 & 3.749 & 1.907 & - & 0.419 & -0.014 \\
\hline 40 & 1.120 & 0.032 & 0.117 & 1.134 & 7.756 & -2.714 & 140.113 & -12.12 & 5.882 & 0.306 & 0.430 & 0.029 \\
\hline 41 & 1.790 & -0.779 & 0.117 & 5.718 & 8.178 & 1.171 & 121.332 & 55.29 & 5.709 & 0.442 & 0.272 & 0.191 \\
\hline 42 & 1.231 & -0.120 & 0.117 & 5.889 & 9.748 & -2.617 & 241.212 & -84.30 & 2.448 & - & 0.365 & 0.163 \\
\hline 43 & 0.909 & 0.011 & 0.117 & 6.559 & 7.668 & 5.514 & 91.187 & 170.0 & 4.372 & - & 0.236 & 0.220 \\
\hline 44 & 0.679 & 0.012 & \begin{tabular}{|l|l|}
0.117 \\
\end{tabular} & 7.700 & 5.361 & 1.227 & 317.360 & 14.53 & 3.635 & 0.169 & 0.169 & -0.001 \\
\hline 45 & 1.017 & 0.064 & 0.117 & 1.234 & 8.287 & -0.032 & 121.417 & 9.892 & 3.381 & - & 0.286 & 0.018 \\
\hline 46 & 0.552 & -0.052 & 0.117 & 3.186 & 4.863 & 9.765 & 131.618 & 0.439 & 3.160 & - & 0.479 & 0.008 \\
\hline 47 & 1.229 & 0.022 & 0.117 & 1.234 & 4.811 & 10.49 & 143.593 & -6.272 & 3.418 & - & 0.480 & 0.004 \\
\hline 48 & 0.600 & 0.521 & \begin{tabular}{|l|l|}
0.117 \\
\end{tabular} & 2.005 & 5.780 & 4.051 & 198.158 & -105.8 & 4.469 & - & 0.420 & -0.205 \\
\hline 49 & 2.071 & -0.029 & 0.117 & 2.555 & 7.536 & 3.991 & 171.469 & -21.01 & 1.601 & - & 0.593 & 0.015 \\
\hline 50 & 2.056 & 0.054 & 0.117 & 1.084 & 9.767 & 2.903 & 174.841 & -20.97 & 2.047 & 0.457 & 0.626 & 0.075 \\
\hline 51 & 1.892 & -0.060 & 0.117 & 3.556 & 5.724 & 1.050 & 125.770 & -31.69 & 6.347 & 0.548 & 0.342 & 0.072 \\
\hline 52 & 1.173 & -0.022 & 0.117 & 0.884 & 11.20 & 2.195 & 113.850 & -2.695 & 5.702 & 0.217 & 0.064 & -0.014 \\
\hline 53 & 0.902 & -0.011 & \begin{tabular}{|l|l|}
0.117 \\
\end{tabular} & 0.133 & 5.796 & 2.634 & 195.398 & -1.892 & 5.884 & \begin{tabular}{|l|l|}
0.214 \\
\end{tabular} & 0.340 & 0.019 \\
\hline 54 & 1.375 & 0.617 & 0.117 & 1.234 & 8.640 & 1.954 & 82.749 & 0.608 & 0.626 & 3.742 & 0.499 & -0.033 \\
\hline 55 & 0.757 & 1.326 & 0.117 & 0.053 & 4.642 & 5.535 & 212.670 & -135.8 & 1.201 & 3.342 & 0.786 & -0.426 \\
\hline
\end{tabular}


Zirconium Diboride-Silicon Carbide Composites

\begin{tabular}{|c|c|c|c|c|c|c|c|c|c|c|c|c|}
\hline Ex.No & MRR & P.E & TWR & P.E & Roughness & P.E & Roundness & P.E & Taperangle & P.E & Overcut & P.E \\
\hline 56 & 0.506 & 0.615 & 0.117 & 1.885 & 13.16 & -3.348 & 58.100 & 28.56 & 6.384 & - & 0.158 & -0.096 \\
\hline 57 & 2.067 & -0.035 & 0.117 & 0.463 & 4.859 & 9.396 & 205.186 & 7.199 & 0.361 & - & 0.383 & 0.033 \\
\hline 58 & 1.514 & -0.392 & 0.117 & 5.718 & 5.043 & 1.059 & 285.685 & -250.9 & 3.131 & - & 0.561 & -0.279 \\
\hline 59 & 0.311 & -0.021 & 0.117 & 3.556 & 9.840 & -3.122 & 164.371 & -6.912 & 3.481 & 0.190 & 0.503 & 0.027 \\
\hline 60 & 1.897 & 0.105 & 0.117 & 1.905 & 4.942 & 1.439 & 111.147 & -1.701 & 4.090 & 0.591 & 0.337 & -0.008 \\
\hline
\end{tabular}

\section{Influences of parameters on the machinability behaviour of the composite}

One of the best techniques to optimize quality characteristics is the desirability function. It is defined as a function which transforms a set of properties into a single objective called "desirability". The global knit quality index is calculated using the desirability function, an individual index " $d_{i}$ " measuring the requirement degree of each property. This index, varying between 0 corresponding to a null satisfaction, and 1 corresponding to total requirements [5], depends on the following three factors:-,

1. The objective given to each property in the global knit quality definition such as maximize, minimize, or reaching a target value.

2. The acceptance interval $[B, A]$ defining the lower and upper limits for each property in which the requirement is satisfied and the value of $\mathrm{s}$ is chosen by the investigator.

3. The consumer requirement level.

In this study, minimization of outputs like roughness, roundness, tool wear rate, taper angle, and overcut has to be carried out. These responses are normalized using equation 11 . The objective function of material removal rate is maximization, so the value of MRR is normalized using equation 12.

$$
\begin{aligned}
& d_{r}^{\min }=\left\{\begin{array}{cc}
0 & \text { if } f_{r}(X)>B \\
\left(\frac{f_{r(X)-B}}{A-B}\right)^{s} & \text { if } A \leq f_{r}(X) \leq B \\
1 & \text { if } f_{r}(X)<A
\end{array}\right. \\
& d_{r}^{\min }=\left\{\begin{array}{cc}
0 & \text { if } f_{r}(X)<A \\
\left(\frac{f_{r(X)-A}}{B-A}\right)^{s} & \text { if } A \leq f_{r}(X) \leq B \\
1 & \text { if } f_{r}(X)>B
\end{array}\right.
\end{aligned}
$$

In this case, the weights are assigned equally. Since the $r$ desirability functions $d_{1} \ldots \ldots d_{r}$ are on the $[0,1]$ scale, they can be combined to achieve the global desirability function (D). One method of doing this is by using the geometric mean

$$
D=\left(\prod_{r=1}^{R} d_{r}{ }^{w_{r}}\right)^{\frac{1}{\sum_{r=1}^{R} w_{r}}}
$$

The geometric mean has the property of showing that if any one model is undesirable $\left(d_{r}=0\right)$, the global desirability is also unacceptable $(D=0)$. Once $D$ has been defined and the prediction equations for each of the $r$ equations have been computed, the global desirability can be used to optimize or rank the predictors. These functions are on the same scale and are discontinuous at the points $A, B$. The values of $s$ can be chosen so that the desirability criterion is easier or more difficult to satisfy. All the responses are given equal weightage of 0.16666 . Global desirability $(D)$ values of all the experiments are calculated by substituting ' $w$ ' values in the equation (13). The tools are ranked based on the global desirability values with equal weights. Figure 5 presents the main effect plot drawn by taking the average of global 
Desirability value corresponding to each tool, pulse on time, pulse off time and workpiece. This plot shows that GR is the best tool and the workpiece with $25 \% \mathrm{SiC}$ is the best workpiece. This plot also shows that the pulse on time of $7 \mu$ s and the pulse off time of $1 \mu$ s are the best values.

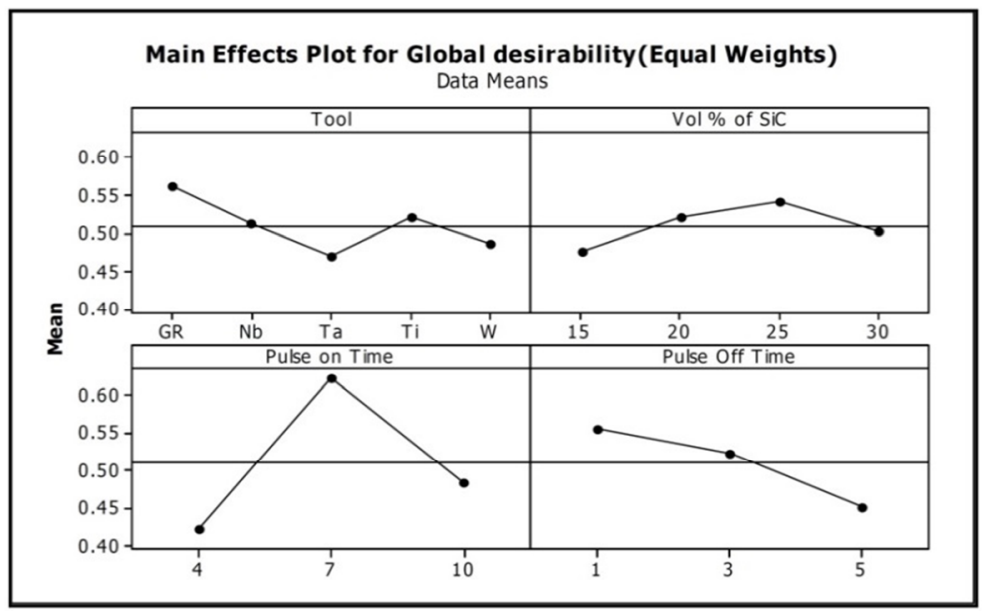

Fig. 5 Main effects plot of global desirability

\section{Conclusion}

DFA confirmed that all the tools, except tantalum, produce a better MRR with the workpiece of $30 \mathrm{vol}$ \% of SiC. Tantalum produces a higher MRR with the workpiece of 25 vol. \% of SiC. Graphite produces a higher MRR with all the workpieces. After graphite, niobium produces the second highest MRR. The feed forward neural network is trained with data samples using the standard back propagation algorithm. Percentage of $\mathrm{SiC}$ in the workpiece material, the product of thermal conductivity and the melting point of the tool material, the pulse on time and the pulse off time are considered as input parameters while MRR, TWR, roughness, roundness, taper angle and overcut are considered as output responses. Using input/output data of the experiments, the model is trained and tested. The network consists of one input layer with four neurons representing the four input parameters and one output layer with six output neurons indicating the six output parameters. The weights are randomly generated for the first iteration. The network is trained using the Levenberg - Marquardt algorithm. The network is trained initially with one neuron in the hidden layer i.e., the 4-4-6 topology is considered for training. The feed forward back propagation neural network model with one hidden layer having 35 neurons is found to be the optimum network model (4-35-35-6). It has the mean correlation coefficient of 0.92408 .

\section{REFERENCES}

[1] Bashiri, M. and Miremadi, S.G., 2014. Performability guarantee for periodic tasks in real-time systems. Scientia Iranica. Transaction D, Computer Science \& Engineering, Electrical, Vol.21, No.6, p.2127, 2014.

[2] V. Cariapa, K.S. Akbay and R. Rudraraju, “Application of neural networks for compliant tool polishing operations", Journal of Materials Processing Technology, Vol. 28, No. 1, Pp. 241-250, 1991. DOI: 10.1016/0924-0136(91)90223-2

[3] J.A. Freeman and D.M. Skapura, "Neural networks: algorithms, applications, and programming techniques", Addison-Wesley, Reading. MA, 1991.

[4] Gjeldum, N., Veza, I., and Bilic, B. "Prediction of the material removal rates of cylindrical wire electrical discharge turning processes." Transactions of Famena, Vol.35, No.1, 2011.

[5] M.S. Hewidy, T.A El Taweel and M.F El Safty, "Modelling the machining parameters of wire electrical discharge machining of Inconel 601 using RSM". Journal of Materials Processing Technology, Vol.169, No.2, Pp.328-336, 2005. DOI: 10.1016/j.jmatprotec.2005.04.078

[6] Y. Kao and Y.S. Tarng, "A neural network approach for the on-line monitoring of the electrical discharge machining process”, Journal of Materials Processing Technology, Vol. 69, No. 1, Pp. 112-119, 1997.

DOI: 10.1016/S0924-0136(97)00004-6 
[7] Karsaj, I., Sansour, C., and Soric, J, "On Modelling of Kinematic Hardening Responses in Finite Strain Elastoplasticity." Transactions of Famena Vol.28, No.2, Pp.1-10, 2004.

[8] Z.Katz and J. Naude, "A neural network/expert system approach for design improvement of products manufactured by EDM”, Journal of Manufacturing science and Engineering: ASME, Vol. 121, No. 4, Pp. 733-738, 1999.

[9] T.J.Ko and D.W. Cho, “Adaptive optimization of face milling operations using neural networks", Journal of Manufacturing Science and Engineering: ASME, Vol.120, Pp. 443-451,1998. DOI: $10.1115 / 1.2830145$

[10] B. Y. Lee, H.S. Liu and Y.S. Tarng, "Modeling and optimization of drilling process", Journal of Materials Processing Technology, Vol.74, No. 1, Pp. 149-157, 1998. DOI: 10.1016/S0924-0136(97)00263-X

[11] Lela, Branimir, Drazen Bajic, and Sonja Jozic, "Regression analysis, support vector machines, and Bayesian neural network approaches to modeling surface roughness in face milling." The International Journal of Advanced Manufacturing Technology, Vol. 42, No.11-12, Pp1082-1088, 2009. DOI: $10.1007 / \mathrm{s} 00170-008-1678-\mathrm{z}$

[12] T.W.Liao and L.J. Chin, "A neural network approach for grinding process: modeling and optimization", International Journal of Machine Tools and Manufacture, Vol. 34, No. 7, Pp. 919-937, 1994. DOI: 10.1016/0890-6955(94)90025-6

[13] J.T.Lina, D. Bhattacharyyaa and V. Kecman, "Multiple regression and neural networks analyses in composites machining", Composites Science and Technology, Vol. 63, No. 3, Pp. 539-548, 2003. DOI: $10.1016 / \mathrm{S} 0266-3538(02) 00232-4$

[14] H.S.Liu and Y.S. Tarng, "Monitoring of the electrical discharge machining process by adductive networks", International Journal of Advanced Manufacturing Technology, Vol. 13, No. 4, Pp. 264-270, 1997. DOI: 10.1007/BF01179608

[15] S.Rangwala and D. Dornfeld, "Sensor integration using neural networks for intelligent tool condition monitoring", Journal of Engineering for Industry: ASME, Vol. 112, No.3, Pp. 219-228, 1999. DOI: $10.1115 / 1.2899578$

[16] Schneider, Daniel Rolph, and Zeljko Bogdan. "Model simulations of pollutant reduction in heavy fuel oil fired furnace." Transactions of Famena, Vol. 26, No.1, Pp.15-26, 2002.

[17] S.Sivasankar, R. Jeyapaul, S. Kolappan, and N. M. Shaadil. "Procedural study for roughness, roundness and waviness measurement of EDM drilled holes using image processing technology." Computer Model Information Proces, Vol. 16,Pp. 49-63, 2012.

[18] Y.S.Tarng, Y.W. Hseih and S.T. Hwang, "Sensing tool breakage in face milling with a neural network", International Journal of Machine Tools and Manufacture, Vol. 34, No.3, Pp. 341-350, 1994. DOI: 10.1016/0890-6955(94)90004-3

[19] Y.S.Tarng, S.T. Hwang and Y.S. Wang, "A neural network controller for constant turning force", International Journal of Machine Tools and Manufacture. Vol. 34, No.4, Pp. 453-460, 1994. DOI: 10.1016/0890-6955(94)90077-9

[20] Y.S.Tarng, S.C. Ma and L.K. Chung, "Determination of optimal cutting parameters in wire electrical discharge machining”, International Journal of Machine Tools and Manufacture, Vol. 35, No. 2, Pp. 1693-1701,1995. DOI: 10.1016/0890-6955(95)00019-T

[21] Urevc, Janez, Pino Koc, and Boris stok. "Characterization of material parameters used in the mathematical modelling of arc welding and heat treatment processes." Transactions of famena, Vol.35, No.4, 2011.

[22] Wilson and R. Sharda, "Neural Networks. OR/MS TODAY", A Joint Publication of ORSA and TIMS, Vol. 19, No. 4, Pp. 36-42, 1992.

[23] Zahedi, "An introduction to neural networks and a comparison with artificial intelligence and expert systems", Interfaces, Vol. 21, No.2, Pp. 25-38, 1991 DOI: 10.1287/inte.21.2.25

[24] Zuperl, Uros, Franc Cus, and Marko Reibenschuh. "Modeling and adaptive force control of milling by using artificial techniques." Journal of Intelligent Manufacturing, Vol.23, No. 5, Pp 1805-1815, 2012. DOI: $10.1007 / \mathrm{s} 10845-010-0487-\mathrm{z}$

Submitted: $\quad 15.08 .2015$

Accepted: $\quad 20.9 .2016$
S.Sivasankar, Assistant Professor, Department of Mechanical Engineering, Government College of Engineering, Sengippatti, Thanjavur. email:shivasankar09@gmail.com R.Jeyapaul, Associate professor, National Institute of Technology, Thiruchirappalli. email:jeyapaul@nitt.edu 